\title{
Apoptosis-mediated antiproliferative activity of friedolanostane triterpenoid isolated from the leaves of Garcinia celebica against MCF-7 human breast cancer cell lines
}

\author{
ANAS SUBARNAS ${ }^{1}$, AJENG DIANTINI $^{1}$, RIZKY ABDULAH $^{1,2}$, ADE ZUHROTUN $^{3}$, \\ PATRIA A. NUGRAHA ${ }^{1}$, YUNI E. HADISAPUTRI ${ }^{4}$, IRMA M. PUSPITASARI ${ }^{1,2}$, \\ CHIHO YAMAZAKI ${ }^{2}$, HIROYUKI KUWANO ${ }^{4}$ and HIROSHI KOYAMA ${ }^{2}$
}

\begin{abstract}
${ }^{1}$ Department of Pharmacology and Clinical Pharmacy, Faculty of Pharmacy, Universitas Padjadjaran, Jatinangor 45363, Indonesia; ${ }^{2}$ Department of Public Health, Gunma University Graduate School of Medicine, Gunma 371-8511, Japan;

${ }^{3}$ Department of Biological Pharmacy, Faculty of Pharmacy, Universitas Padjadjaran, Jatinangor 45363, Indonesia;

${ }^{4}$ Department of General Surgical Science, Gunma University Graduate School of Medicine, Gunma 371-8511, Japan
\end{abstract}

Received July 31, 2015; Accepted October 2, 2015

DOI: $10.3892 /$ br. 2015.532

\begin{abstract}
The leaves of Garcinia celebica strongly inhibit the proliferation of MCF-7 human breast adenocarcinoma cell lines. The present study focused on investigating the active anticancer and antiproliferative compound from the G. celebica leaves and assessing its mechanism of action. Ethanol extracts of $G$. celebica were fractionated based on their polarity using $n$-hexane, ethyl acetate and water. The antiproliferative properties were tested in vitro against MCF-7 human breast cancer cell lines using the 3-(4,5-dimethylthiazolyl-2)-2,5-diphenyltetrazolium bromide assay. The active compound was subsequently isolated using column chromatography and identified by nuclear magnetic resonance. The characterized compound was also tested for its antiproliferative properties and the mechanism by which it induces apoptosis in MCF-7 cells by western blot analysis of the activated apoptotic proteins. This resulted in the isolation of a friedolanostane triterpenoid, which was determined to be methyl-3 $\alpha$, 23-dihydroxy-17,14-friedolanstan-8,14,24-trien-26-oat. This compound inhibited MCF-7 cell proliferation in a time- and dose-dependent manner with $\mathrm{IC}_{50}$ values of 82 and $70 \mu \mathrm{M}$ for the 24 and $48 \mathrm{~h}$ treatments, respectively. Furthermore, the western blot analysis suggested that the compound exerted its anticancer activities by promoting apoptosis through the inhibition of the oncogenic protein Akt, thereby increasing the expression of poly (ADP-ribose) polymerase (PARP)
\end{abstract}

Correspondence to: Professor Anas Subarnas, Department of Pharmacology and Clinical Pharmacy, Faculty of Pharmacy, Universitas Padjadjaran, J1. Raya Bandung Sumedang KM 21, Jatinangor 45363, Indonesia

E-mail: aasubarnas@yahoo.co.id

Key words: Garcinia celebica, primates, cancer, apoptosis protein. These results suggest that methyl-3 $\alpha, 23$-dihydrox y-17,14-friedolanstan-8,14,24-trien-26-oat is the anticancer compound found in G. celebica, providing a basis for its potential use in cancer disease management.

\section{Introduction}

Cancer remains an extremely serious life-threatening disease for all humans. Although continuous efforts have been made to provide novel leads against cancers, and numerous cancer drugs have been derived from plants or generated synthetically, the current drugs used clinically have no significant effectiveness or safety (1). Therefore, it is important to undertake research into the discovery of new anticancer drugs of plant origins. Numerous types of bioactive compounds have been isolated from medicinal plants and several of these compounds are currently undergoing further investigation (2).

As plants consumed by primates are assumed to be a promising source of therapeutic agents for human disease management, a series of studies have been conducted to search for anticancer agents from plant sources with a focus on finding new potential drugs or leads for breast cancer from primate-consumed plants (3). Breast cancer is the most malignant form of cancer among women, causing $>1.2$ million new cases and 0.5 million mortalities annually (4). Our previous studies revealed that kaempferol-3-O-rhamnoside, isolated from the leaves of Schima walichii Korth, a plant commonly consumed by primates, inhibited the proliferation of the MCF-7 breast cancer cell line through activation of the caspase cascade pathway (5). Furthermore, an evaluation of 42 species of Indonesian primate-consumed plants revealed that several plant extracts, including the $n$-hexane fraction of the Garcinia celebica (G. celebica) leaves extract, had potent antiproliferative activity against MCF-7 cells (6). In the present study, a compound from the $n$-hexane fraction of the G. celebica leaves extract with antiproliferative activity against MCF-7 cell lines was identified and the pro-apoptotic activity of the active compound was evaluated. 


\section{Materials and methods}

Collection of plant materials. Leaves of G. celebica were collected in the Pangandaran Beach Conservation Area of West Java (Indonesia). The plant species was identified by the Department of Biology, Faculty of Mathematics and Natural Sciences, Universitas Padjadjaran (Jatinangor, Indonesia). The leaves were dried in the open air away from direct sunlight.

Isolation of an active compound. Dried G. celebica leaves were powdered and extracted with 95\% ethanol (three times every $24 \mathrm{~h}$ ) at room temperature and the solvent was evaporated under reduced pressure at $50^{\circ} \mathrm{C}$ to yield concentrated extracts. The extract was partitioned with a mixture of $n$-hexane-water $(3: 1)$ to generate hexane and water layers. The water layer was further extracted with ethyl acetate to yield ethyl acetate and water fractions. The $n$-hexane and ethyl acetate fractions had inhibitory activities against MCF-7 cell proliferation. Silica gel chromatography was used to elute the $n$-hexane fraction $(23.32 \mathrm{~g})$ into 5 fractions of $n$-hexane-ethyl acetate mixtures with increasing polarity ( $n$-hexane:ethyl acetate, 9:1, 8:2, 7:3, 6:4, 5:5). Each fraction was tested for its toxicity against $A$. salina larvae (7), and the fraction with the highest toxicity was further chromatographed over silica gel with the same elution system to generate 11 fractions, of which fraction 6 contained the targeted compounds. Fraction 6 was subjected to preparative thin-layer chromatography using a mixture of chloroform-methanol (9.5:05) as a developing solvent system. The application of preparative chromatography resulted in a compound $(50.20 \mathrm{mg}$, white amorphous solid) that showed high toxicity against A. salina larvae. The compound was identified by an analysis of its spectroscopic data [ultraviolet (UV), infrared (IR), mass spectrometry (MS) and nuclear magnetic resonance (NMR)].

Cell culture and drug-sensitivity assays. MCF-7 human breast cancer cell lines were purchased from the American Type Culture Collection (Manassas, VA, USA). The cell lines were cultured in RPMI-1640 medium (Sigma-Aldrich, St. Louis, MO, USA) supplemented with $10 \%$ fetal bovine serum and antibiotics (100 U/ml penicillin and $100 \mu \mathrm{g} / \mathrm{ml}$ streptomycin). Cell proliferation was analyzed using an 3-(4,5-dimethylthiazolyl-2)-2,5-diphenyltetrazolium bromide (MTT) assay in cells treated with various concentrations of the active compound isolated from leaves of G. celebica following the methods of Abdulah et al (8). Briefly, $2 \times 10^{4}$ cells in $50 \mu \mathrm{l} /$ well were plated in 96-well plates. Following the initial cell seeding, different concentrations of the active compound isolated from leaves of G. celebica were added and incubated for $24 \mathrm{~h}$. WST-8 assay cell-counting solution (Dojindo Lab., Kumamoto, Japan) was added to each well $(10 \mu \mathrm{l})$ and incubated at $37^{\circ} \mathrm{C}$ for $3 \mathrm{~h}$. After the addition of $100 \mu \mathrm{l} /$ well of $1 \mathrm{~N} \mathrm{HCl}$, the cell proliferation rate was subsequently determined by measuring the absorbance at a wavelength of $450 \mathrm{~nm}$. The absorbance was read using a microtiter plate reader (Becton-Dickinson, Franklin Lakes, NJ, USA).

Cell extraction and western blot analysis. Protein concentrations were determined using a bicinchoninic acid protein assay kit (Pierce Biotechnology, Inc., Rockford, IL, USA). In total, $40 \mu \mathrm{g}$ protein was electrophoresed on a Mini-PROTEAN TGX
Precast gel (4-20\%; Bio-Rad Laboratories, Hercules, CA, USA) and electrotransferred to a $7 \times 8 \mathrm{~cm}$ Hybond-enhanced chemiluminescence membrane (GE Healthcare Life Sciences, Little Chalfont, UK). Apoptosis-associated proteins were analyzed by immunoblot analysis using poly (adenosine diphosphate-ribose) polymerase (PARP; \#9542) and Akt (\#4685) antibodies at a 1:1,000 dilution (both Cell Signaling Technology, Danvers, MA, USA). $\beta$-actin (\#A5441; Sigma-Aldrich) served as the loading control.

\section{Results}

Structural determination of methyl-3 $\alpha, 23$-dihydroxy-17,14friedolanstan-8,14,24-trien-26-oat from G. celebica leaves. An antiproliferative compound isolated from the leaves of G. celebica was amorphous and was white in color. It exhibited a molecular ion peak at $\mathrm{m} / \mathrm{z} 484$ in the electron ionization mass spectrum indicating that this compound had a molecular formula of $\mathrm{C}_{31} \mathrm{H}_{48} \mathrm{O}_{4}$.

The UV spectrum indicated absorption bands at 275 and $439 \mathrm{~nm}$ and the IR spectrum revealed the presence of a hydroxyl group (3,500 $\left.\mathrm{cm}^{-1}\right)$ and a carbonyl group of an $\alpha, \beta$-unsaturated ester $\left(1,700 \mathrm{~cm}^{-1}\right)$. The presence of the carbonyl functionality was further confirmed by a carbon signal appearing at $\delta 168.7$ in the ${ }^{13} \mathrm{C}$ NMR spectrum.

The ${ }^{1} \mathrm{H}$ and ${ }^{13} \mathrm{C}$ NMR spectra of the compound exhibited that this compound was assumed to be a triterpenoid compound. The ${ }^{1} \mathrm{H}$ NMR spectrum indicated the presence of 5 tertiary methyls ( $\delta 0.76,0.89,0.90,0.98$ and 1.01$), 1$ secondary methyl $(\delta 0.94, d, \mathrm{~J}=7.5 \mathrm{~Hz})$, and 1 oxymethine proton $(\delta 3.45, b r s)$. These signals were assumed due to a tetracyclic triterpene having a $3 \alpha$-hydroxy group (9-11). Furthermore, signals due to 1 olefinic proton $(\delta 6.72, q d, \mathrm{~J}=7.5$ and $1.5 \mathrm{~Hz}), 1$ vinylic methyl $(\delta 1.87, d, \mathrm{~J}=1.5 \mathrm{~Hz}), 1$ oxymethine proton $(\delta 4.57$, $d d d, \mathrm{~J}=10.7,7.5$ and $2.5 \mathrm{~Hz}$ ), and methoxy protons $(\delta 3.75, s)$ were observed. These signals suggested that the compound had a side chain with a structure of $\left[-\mathrm{CH}(\mathrm{Me}) \mathrm{CH}_{2} \mathrm{CH}(\mathrm{OH})\right.$ $\left.\mathrm{CH}=\mathrm{C}(\mathrm{Me}) \mathrm{COOCH}_{3}\right]$. The ${ }^{13} \mathrm{C}$ NMR spectrum indicated the presence of 7 methyl carbons $(\delta 12.9,15.5,15.8,17.3$, $19.2,22.4$ and 28.2), 8 methylene carbons ( $\delta$ 18.3, 22.9, 25.8, $26.9,29.4,30.3,39.7$ and 45.7), 6 methine carbons ( $\delta 33.6$, $44.6,67.1,76.0,116.0$ and 142.6), and 9 quartenary carbons (o 37.8, 38.0, 48.2, 50.2, 123.1, 127.3, 144.6, 148.9 and 168.7). These NMR spectral data showed that the compound may be friedolanostane triterpenoid with one tetrasubstituted double bond and two trisubstituted double bonds. One of the two trisubstituted double bonds was located in the side chain and the two remaining double bonds in the tetracyclic system were indicated to be conjugated. The conjugated double bonds were assumed to be present at C-8/C-9 and C-14/C-15, therefore, the vinylic proton appearing at $5.26(s)$ was at C-15. Thus, the structure of the compound was established as methyl- $3 \alpha$, 23-dihydroxy-17,14-friedolanstan-8,14,24-trien-26-oat (Fig. 1) which has been reported previously. The ${ }^{1} \mathrm{H}$ and ${ }^{13} \mathrm{C}$ NMR data of the identified compound is shown in Table I. Confirmation of the structure was obtained by comparison of its spectral data with those reported in previous studies $(11,12)$.

Inhibitory activity of methyl-3 $\alpha, 23$-dihydroxy-17,14-friedolanstan-8,14,24-trien-26-oat against MCF-7 cell line 
Table I. ${ }^{1} \mathrm{H}$ and ${ }^{13} \mathrm{C}$ NMR data of methyl-3 $\alpha, 23$-dihydroxy-17,14friedolanstan-8,14,24-trien-26-oat isolated from $G$. celebica leaves.

\begin{tabular}{|c|c|c|c|c|}
\hline \multirow[b]{2}{*}{ No. } & \multicolumn{2}{|c|}{${ }^{13} \mathrm{C}$ NMR } & \multicolumn{2}{|r|}{${ }^{1} \mathrm{H}$ NMR } \\
\hline & Chemical shift & Position & Chemical shift & Position \\
\hline 1 & 168.7 & $\mathrm{C}-26$ & & \\
\hline 2 & 148.9 & C-14 & & \\
\hline 3 & 144.6 & C-9 & & \\
\hline 4 & 142.6 & $\mathrm{C}-24$ & 6.72 & $\begin{array}{l}\mathrm{H}-24(1 \mathrm{H}, q d, \mathrm{~J}=7.5 \& \\
1.5 \mathrm{~Hz})\end{array}$ \\
\hline 5 & 127.3 & $\mathrm{C}-25$ & & \\
\hline 6 & 123.1 & C-8 & & \\
\hline 7 & 116.0 & C-15 & 5.26 & $\mathrm{H}-15(1 \mathrm{H}, s)$ \\
\hline 8 & 76.0 & $\mathrm{C}-3$ & 3.45 & $\mathrm{H}-3(1 \mathrm{H}, b r s)$ \\
\hline 9 & 67.1 & $\mathrm{C}-23$ & 4.57 & $\begin{array}{l}\mathrm{H}-23(1 \mathrm{H}, d d d, \mathrm{~J}=10.7 \\
7.5 \& 2.5 \mathrm{~Hz})\end{array}$ \\
\hline 10 & 52.1 & $\mathrm{OMe}$ & 3.75 & $\mathrm{OMe}(3 \mathrm{H}, s)$ \\
\hline 11 & 50.2 & $\mathrm{C}-17$ & & \\
\hline 12 & 48.2 & $\mathrm{C}-13$ & & \\
\hline 13 & 45.7 & C-16 & $\begin{array}{l}2.23-2.15 \\
1.99-1.94\end{array}$ & $\begin{array}{l}\mathrm{H}-16, \mathrm{H}-20(2 \mathrm{H}, m) \\
\mathrm{H}-16(1 \mathrm{H}, m)\end{array}$ \\
\hline 14 & 44.6 & $C-5$ & & \\
\hline 15 & 39.7 & $\mathrm{C}-11$ & & \\
\hline 16 & 38.0 & $\mathrm{C}-10$ & & \\
\hline 17 & 37.8 & $\mathrm{C}-4$ & & \\
\hline 18 & 33.6 & $\mathrm{C}-20$ & & \\
\hline 19 & 30.3 & $\mathrm{C}-1$ & $1.77-1.56$ & $\begin{array}{l}2 \times \mathrm{H}-1, \mathrm{H} 2, \mathrm{H} 5, \\
2 \mathrm{x} \mathrm{H}-6,2 \mathrm{x}-12, \mathrm{H}-11 \\
(9 \mathrm{H}, m)\end{array}$ \\
\hline 20 & 29.4 & $\mathrm{C}-2$ & $1.16-1.10$ & $\mathrm{H}-2, \mathrm{H}-11(2 \mathrm{H}, m)$ \\
\hline 21 & 28.2 & C-28 & 0.98 & $\mathrm{Me}-28(3 \mathrm{H}, s)$ \\
\hline 22 & 26.9 & $\mathrm{C}-7$ & $2.37-2.31$ & $\mathrm{H}-7, \mathrm{H}-7(2 \mathrm{H}, m)$ \\
\hline 23 & 25.8 & $\mathrm{C}-12$ & & \\
\hline 24 & 22.9 & $\mathrm{C}-22$ & $2.08-2.05$ & $2 \times \mathrm{H}-22(2 \mathrm{H}, m)$ \\
\hline 25 & 22.4 & $\mathrm{C}-29$ & 0.89 & $\mathrm{Me}-29(3 \mathrm{H}, s)$ \\
\hline 26 & 19.2 & $\mathrm{C}-30$ & 1.01 & $\operatorname{Me}-30(3 \mathrm{H}, s)$ \\
\hline 27 & 18.3 & $C-6$ & & \\
\hline 28 & 17.3 & C-19 & 0.90 & $\mathrm{Me}-19(3 \mathrm{H}, s)$ \\
\hline 29 & 15.8 & C-18 & 0.76 & $\mathrm{Me}-18(3 \mathrm{H}, s)$ \\
\hline 30 & 15.46 & $\mathrm{C}-21$ & 0.94 & $\begin{array}{l}\mathrm{Me}-21(3 \mathrm{H}, d, \\
\mathrm{J}=7.5 \mathrm{~Hz})\end{array}$ \\
\hline 31 & 12.93 & $\mathrm{C}-27$ & 1.87 & $\begin{array}{l}\mathrm{Me}-27(3 \mathrm{H}, d \\
\mathrm{J}=1.5 \mathrm{~Hz})\end{array}$ \\
\hline
\end{tabular}

proliferation. Methyl-3 $\alpha, 23$-dihydroxy-17,14-friedolanstan-8, 14,24-trien-26-oat was evaluated for its effect on the proliferation of MCF-7 breast cancer cell lines by the MTT assay. The evaluation resulted in a time- and dose-dependent manner inhibition of the compound on the cell proliferation (Fig. 2). The compound strongly inhibited the MCF-7 cell line proliferation in 24 and $48 \mathrm{~h}$ treatments, with the $\mathrm{IC}_{50}$ value of 82 and $70 \mu \mathrm{M}$ in the 24 and $48 \mathrm{~h}$ measurements, respectively.

Proapoptotic activity of methyl-3 $\alpha, 23$-dihydroxy-17,14friedolanstan-8,14,24-trien-26-oat through PARP protein activation. The MTT assay showed strong inhibitory activity of methyl-3 $\alpha, 23$-dihydroxy-17,14-frie-

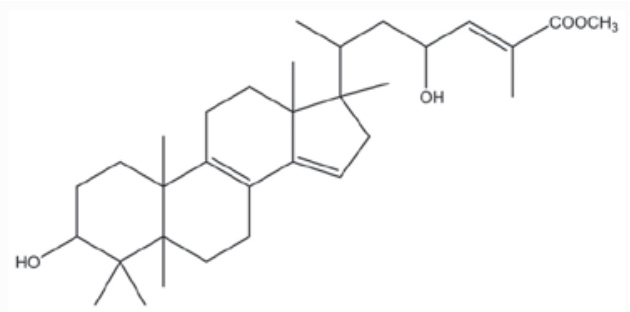

Figure 1. Methyl-3 $\alpha, 23$-dihydroxy-17,14-friedolanstan-8,14,24-trien-26-oat isolated from G. celebica leaves.

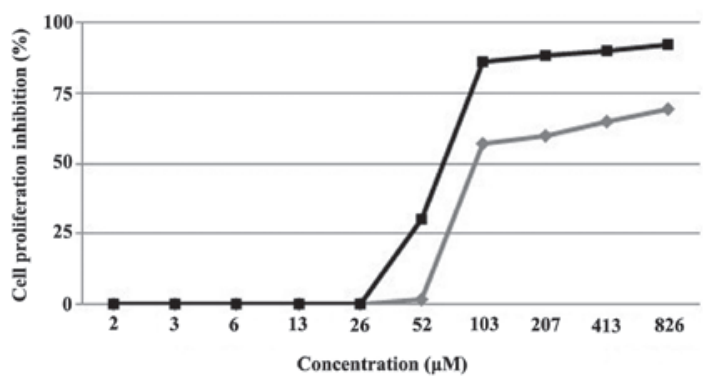

$\multimap 24 \mathrm{~h} \rightarrow-48 \mathrm{~h}$

Figure 2. Effects of 24 and $48 \mathrm{~h}$ treatments of methyl-3 $\alpha, 23$-dihydroxy-17, 14-friedolanstan-8,14,24-trien-26-oat obtained from the G. celebica leaves on the proliferation of MCF-7 breast cancer cell lines.

methyl-3 $\alpha, 23$-dihudroxy-17,14-friedolanstan-8,14,24-trien-26-oat

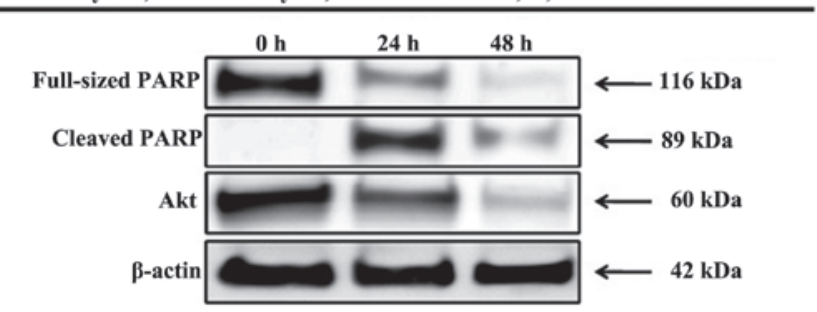

Figure 3. Pro-apoptotic activity of methyl-3 $\alpha, 23$-dihydroxy-17,14-friedolanstan8,14,24-trien-26-oat obtained from the G. celebica leaves.

dolanstan-8,14,24-trien-26-oat against the MCF-7 cell line proliferation, thus, the compound was further examined for its proapoptotic activity through PARP protein activation within 24 and $48 \mathrm{~h}$ of treatment. The inhibition of MCF-7 human breast cancer cells proliferation by the compound was mediated by the induction of apoptosis, marked by PARP protein activation, which is one of the most optimal biomarkers of apoptosis. Furthermore, the compound also inhibited the expression of Akt oncogene (Fig. 3).

\section{Discussion}

Traditional medicinal plants have long been regarded as a source of potential therapeutic agents, and the search for new drugs is usually based on this approach (13). In drug discovery, our previous studies recently applied a new approach of selecting plants based on the use of those plants by primates $(3,5)$. In our previous study, the extracts of the G. celebica leaves were strongly cytotoxic to the MCF-7 breast 
cancer cell line (6). Thus, these extracts had the potential for further investigation.

The present study was focused on identifying an antiproliferative compound from the G. celebica leaves. This study resulted in the isolation of a friedolanostane triterpenoid, methyl-3 $\alpha$, 23-dihydroxy-17,14-friedolanstan-8,14,24-trien-26-oat, which strongly inhibited the MCF-7 cell line proliferation in a timeand dose-dependent manner, with $\mathrm{IC}_{50}$ values of 82 and $70 \mu \mathrm{M}$ in the 24 and $48 \mathrm{~h}$ treatments, respectively.

This compound has not been reported previously in connection with its cytotoxicity in these cancer cell lines. Through investigating the anticancer activities of Garcinia plants, numerous studies have focused on xanthone derivatives, which are the main compounds of any Garcinia species (14-16). These findings will thus be valuable supporting information for revealing the efficacy of Garcinia species as a potential anticancer source.

In the present study, this compound was also found to induce the apoptosis of MCF-7 cells, which was indicated by the changes in the expression levels of PARP. The expression levels of PARP were analyzed after 24 and $48 \mathrm{~h}$ of treatment. The N-terminal fragment of PARP, which is an $89-\mathrm{kDa}$ peptide cleaved from full-length PARP $(116 \mathrm{kDa})$, was detected as early as $24 \mathrm{~h}$ after treatment of the MCF-7 cells with methyl-3 $\alpha$, 23-dihydroxy-17,14-friedolanstan-8,14,24-trien-26-oat. Akt, as one of the most important survival signaling pathways in malignancy, has an important role in determining the chemosensitivity of cancer cells. These survival signaling proteins were decreased by treatment with methyl-3 $\alpha, 23$-dihydroxy-17,14-friedolanstan-8,14,24-trien-26-oat, as shown by the reduced expression of the Akt protein.

However, the present study is not without limitations. A detailed mechanism regarding the effect of methyl-3 $\alpha, 23$ dihydroxy-17,14-friedolanstan-8,14,24-trien-26-oat on the expression of several proteins that may directly be affected were not performed, including pro- or anti-apoptotic, estrogen receptor $\alpha$ and phosphorylated Akt (Ser473) proteins.

In conclusion, the present results suggest that methyl-3 $\alpha, 23$ dihydroxy-17,14-friedolanstan-8,14,24-trien-26-oat inhibited the growth of MCF-7 cells through the induction of apoptosis and downregulation of the oncogene Akt. Further evaluation of its toxicity and detailed mechanisms of its antiproliferative action is required to provide a scientific basis for its chemopreventive and chemotherapeutic application in breast cancer management.

\section{Acknowledgements}

The present study was financially supported by the Directorate General of Higher Education of the Ministry of
Education and Culture of Indonesia (Grand-in-Aid for the International Research Collaborations and Publications; grant no. 430/SP2H/PP/DP2 M/VII/2010) for AS.

\section{References}

1. Sakarkar DM and Deshmukh VN: Ethnopharmacological review of traditional medicinal plants for anticancer activity. Int J Pharm Tech Res 3: 298-308, 2011.

2. Kinghorn AD, Chai HB, Sung CK and Keller WJ: The classical drug discovery approach to defining bioactive constituents of botanicals. Fitoterapia 82: 71-79, 2011.

3. Koshimizu K, Murakami A, Hayashi H, et al: Biological activities of edible and medicinal plants from Indonesia and Malaysia. In: Proceedings of The Tokyo International Forum on Conservation and Sustainable Use of Tropical Bioresources, Tokyo, pp203-208, 1998.

4. Ferlay J, Shin HR, Bray F, Forman D, Mathers C and Parkin DM: Estimates of worldwide burden of cancer in 2008: GLOBOCAN 2008. Int J Cancer 127: 2893-2917, 2010.

5. Diantini A, Subarnas A, Lestari K, Halimah E, Susilawati Y, Supriyatna, Julaeha E, Achmad TH, Suradji EW, Yamazaki C, et al: Kaempferol-3-O-rhamnoside isolated from the leaves of Schima wallichii Korth. Inhibits MCF-7 breast cancer cell proliferation through activation of the caspase cascade pathway. Oncol Lett 3: 1069-1072, 2012.

6. Subarnas A, Diantini A, Abdulah R, et al: Antiproliferative activity of primates-consumed plants against MCF-7 human breast cancer cell lines. E3 J Med Res 1: 38-43, 2012.

7. Meyer BN, Ferrigni NR, Putnam JE, Jacobsen LB, Nichols DE and McLaughlin JL: Brine shrimp: A convenient general bioassay for active plant constituents. Planta Med 45: 31-34, 1982.

8. Abdulah R, Faried A, Kobayashi K, Yamazaki C, Suradji EW, Ito K, Suzuki K, Murakami M, Kuwano H and Koyama H: Selenium enrichment of broccoli sprout extract increases chemosensitivity and apoptosis of LNCaP prostate cancer cells. BMC Cancer 9: 414, 2009.

9. Shiao MS, Lin LJ and Yeh SF: Triterpenes in Ganoderma lucidum. Phytochemistry 27: 873-875, 1988.

10. Lin LJ, Shiao MS and Yeh SF: Triterpenes from Ganoderma lucidum. Phytochemistry 27: 2269-2271, 1988.

11. Rukachaisirikul V, Adair A, Dampawan P, Taylor WC and Turner PC: Lanostanes and friedolanostanes from the pericarp of Garcinia hombroniana. Phytochemistry 55: 183-188, 2000.

12. Rukachaisirikul V, Saelim S, Karnsomchoke P and Phongpaichit S: Friedolanostanes and lanostanes from the leaves of Garcinia hombroniana. J Nat Prod 68: 1222-1225, 2005.

13. Fabricant DS and Farnsworth NR: The value of plants used in traditional medicine for drug discovery. Environ Health Perspect 109 (Suppl 1): S69-S75, 2001.

14. Matsumoto K, Akao Y, Ohguchi K, Ito T, Tanaka T, Iinuma M and Nozawa Y: Xanthones induce cell-cycle arrest and apoptosis in human colon cancer DLD-1 cells. Bioorg Med Chem 13: 6064-6069, 2005.

15. Suksamrarn S, Komutiban O, Ratananukul P, Chimnoi N, Lartpornmatulee $\mathrm{N}$ and Suksamrarn A: Cytotoxic prenylated xanthones from the young fruit of Garcinia mangostana. Chem Pharm Bull (Tokyo) 54: 301-305, 2006.

16. Akao $Y$, Nakagawa $Y$, Iinuma $M$ and Nozawa $Y$ : Anti-cancer effects of xanthones from pericarps of mangosteen. Int J Mol Sci 9: 355-370, 2008. 